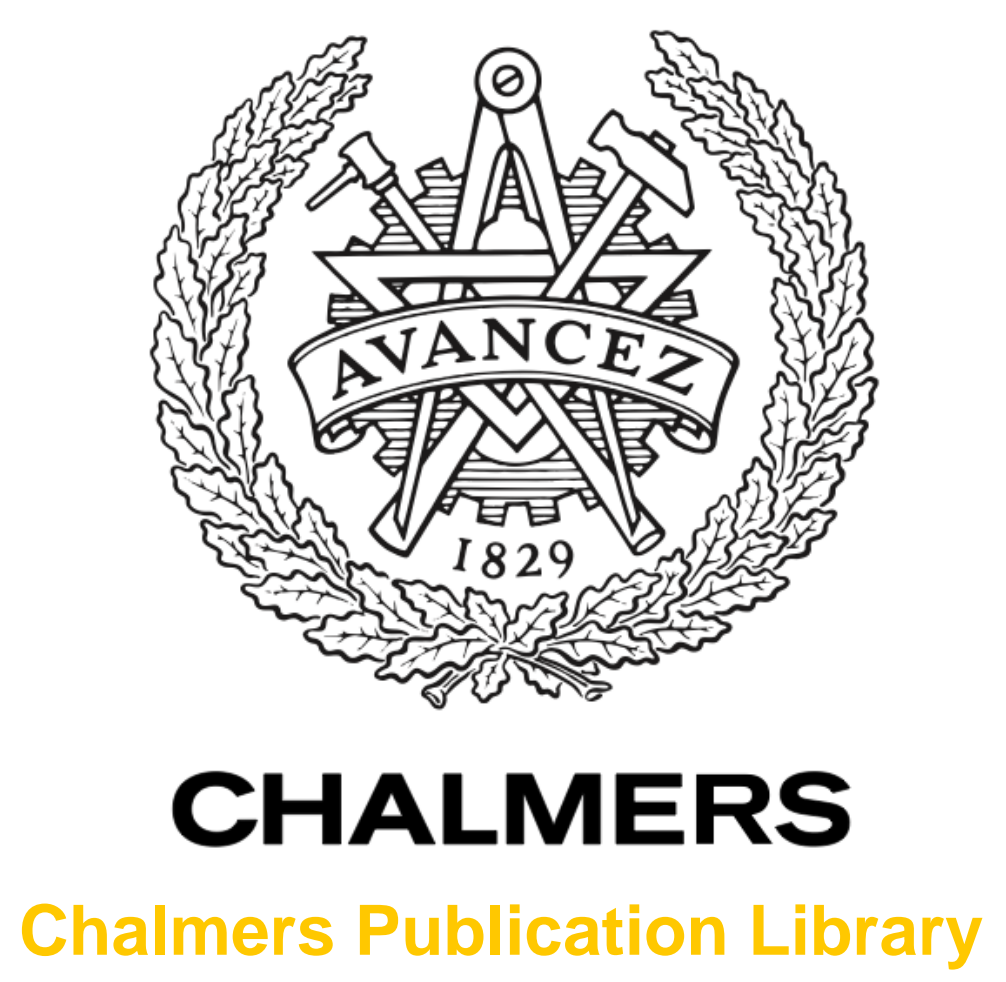

Practical applications of automatic image analysis for overhead lines

This document has been downloaded from Chalmers Publication Library $(\mathrm{CPL})$. It is the author's version of a work that was accepted for publication in:

22nd International Conference \& exhibition on Electricity Distribution (CIRED 2013), Stockholm, 10-13 June, 2013

Citation for the published paper:

Gu, I. ; Berlijn, S. ; Gutman, I. (2013) "Practical applications of automatic image analysis for overhead lines". 22nd International Conference \& exhibition on Electricity Distribution

(CIRED 2013), Stockholm, 10-13 June, 2013 pp. 4 (Session 1, paper 560).

Downloaded from: http://publications.lib.chalmers.se/publication/176693

Notice: Changes introduced as a result of publishing processes such as copy-editing and formatting may not be reflected in this document. For a definitive version of this work, please refer to the published source. Please note that access to the published version might require a subscription. 


\section{PRACTICAL APPLICATIONS OF AUTOMATIC IMAGE ANALYSIS FOR OVERHEAD LINES}

\author{
Irene Y.H. GU \\ Chalmers Univ. of Technology \\ Göteborg, Sweden \\ irenegu@chalmers.se
}

\author{
Sonja BERLIJN \\ Statnett \\ Oslo, Norway \\ sonja.Berlijn@statnett.no
}

\author{
Igor GUTMAN \\ STRI AB \\ Ludvika, Sweden \\ igor.gutman@stri.se
}

\author{
Math H.J.BOLLEN \\ Luleå Univ. of Technology \\ Skellefteå, Sweden \\ math.bollen@1tu.se
}

\begin{abstract}
This paper proposes novel techniques for automatically extracting information from overhead lines based on automatic image/video processing. Three application scenarios are presented: (a) detecting snow and ice coverage on insulators for overhead lines; (b) detecting swing angles of insulators; (c) quantifying the visibility of insulators at transmission lines with typical backgrounds. Experiments have been conducted on recorded videos/images of insulators (with different prototypes) for overhead lines in a remote test station in Norway. Results show that the proposed automatic image analysis methods are promising for extracting the status (snow/ice coverage, swing angle, visibility) of insulators and might be applied in the future for other components of the overhead line.
\end{abstract}

\section{INTRODUCTION}

Automatic monitoring of power system has drawn increasing interests lately, ranging from hourly-meter reading with network users, to power-quality monitoring at important substations through partial-discharge detection of underground medium-voltage cables. Up to now, no automatic image/video processing techniques have been applied to the monitoring/surveillance of power system components, e.g. for extracting information about their status. This paper presents the first of such kind applications by using video surveillance and automatic image/video analysis techniques for extracting information from insulators on overhead lines (OHL). We propose methods related to three applications. In all three cases the methods have been applied successfully to a large set of actual images taken from overhead lines and their components in a test site of Norway.

The first application concerns the detection of snow/ice coverage on insulators (with different profiles) for overhead lines. By obtaining information on snow/ice coverage, a network operator is able to gather experience on how it is accumulated in real outdoor environments. Such information is needed for being able to choose the best suited type of insulators for ice/snow environments.

The second application concerns the detection of swing angles of insulators with respect to the vertical position. High values of the swing angle may lead to an increased failure rate, e.g. during periods of high wind speed. The method has been tested for the same data set as the one for snow and ice coverage.
The third application is to quantify the visibility of insulators for human observers looking at a typical OHL and typical backgrounds (e.g. forest, sky). Low visibility is preferred so as to minimize the visual impact of OHLs. Knowledge on visibility of different insulators and towers is important for designing future OHLs. The aim of image analysis is to avoid subjective visibility inspections as they may vary significantly from person to person, and also to avoid round-clock human inspections. The method has been tested on images captured from the same test site, results show the possibility of automatic quantifying the visibility of insulators by some objective measures.

Some important reasons for automatic image analysis in this study are: to avoid round-clock human inspection and manual evaluations; to develop objective measuring or evaluating methods; to avoid subjective decision of individual person when evaluating many images; to select important events among image scenarios; finally for potential future measuring/monitoring stations.

\section{MEASUREMENTS AND INSULATORS}

A series of investigations of icing and pollution performance of different insulator options have been carried out by Stattnett (the Norwegian transmissionsystem operator) both in laboratory and in the field for comparing different types of insulator and profiles, relating to issues of snow, ice and visibility. This is because icing and pollution are dimensioning environmental parameters in Norway. It is also common knowledge that accumulated snow and ice on insulators may cause flashovers and line outages. Timely and automatic detecting such events would make it possible to prevent possible outages and to choose optimal insulator profiles. Another new issue in designing new OHLs is the visual impact of OHLs, where objective quantification of visibility of different OHLs components against different backgrounds became an interesting issue. Applications of such techniques to OHLs using image processing techniques are a completely new attempt but could become of great importance to Statnett and other utilities in the world facing similar issues. It is important to note that these techniques, when fully developed, have a range of applications (for different line components such as insulators, conductors and towers).

The measurements are performed for insulators on a $420 \mathrm{kV}$ power transmission line, a part of which is converted for remote outdoor tests. Basic components of the monitoring system include visual cameras and lamps, 
a weather station, a communication system between the test site and network operator, a web-based database, and a real-time automatic image analysis system. Measurements (including videos) are done in the remote site (where $230 \mathrm{~V}$ supply is not available) in a fix time interval and during severe conditions (e.g. low temperatures, darkness) where the power is only sufficient for heating the lamps and cameras. Armadillo cameras are used, with Schneider/xenoplan lens of $1.9 / 35 \mathrm{~mm}$, heating is used to avoid moisture on the lens. Three halogen beams with pen beams are used for lighting, each being an effective 75 Watt, equipped with an internal heating to avoid moisture on the glass. Weather and wind conditions are also recorded. Measured data are transferred through the communication system from the test site to the database and stored, and are accessible via a web interface immediately after the measurement [1,2]. Real-time image analysis is performed for each newly captured image and the results are displayed and stored in the system [3].

For measuring the snow/ice, three typical insulators are used in the test site: a composite insulator with a specific profile; a string of glass insulators combining standard and aerodynamic profiles; a string of glass insulator of anti-fog profile, see Fig.1 from left to right for examples of zoomed in insulator images. Images were captured in every 10 minutes.

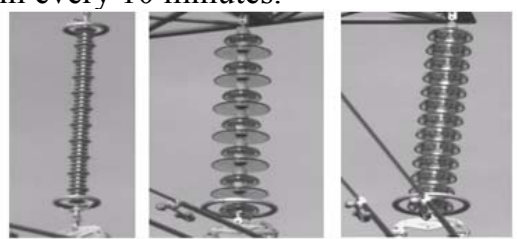

Fig. 1 Zoomed in views of 3 insulator images.

For measuring the visibility of insulators, 3 types of capand-pin insulators are used, and 2 sets of image resolutions (480x640, 960×1280 pixels) are used (see Fig.2). Time interval for capturing video frames is set to 20 minutes.

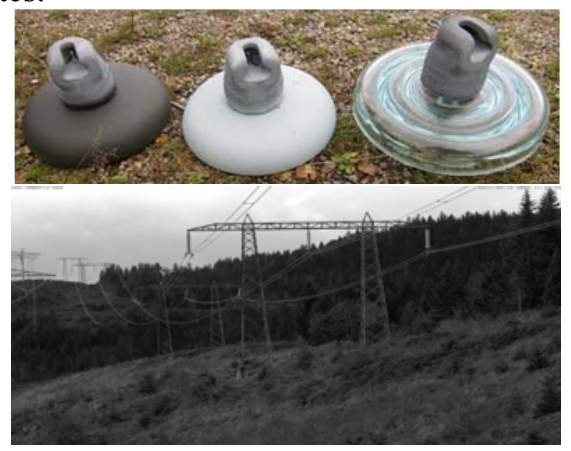

Fig.2 Top: Insulators used for visibility tests. Left to right: olive green RTV-coated; grey RTV-coated; and standard one, all glass insulator string. Bottom: image captured from the test site.

\section{DETECTION AND ESTIMATION OF SNOW/ ICE COVERAGE}

Although insulators are rigid objects and cameras are stationary, automatic detection and analysis of snow coverage is by far a trivial task. Among them, some phenomena that significantly impact the image analysis are:

- Strong wind: cause camera movement hence the insulator position in the image drift;

- Dark weather: lead to low visibility or low contrast in images;

- Clouds: lead to non-uniform fast changing background;

- Fog: may lead to low visibility / severely blurred images;

- Darkness: images vary significantly, depending on the snow, reflection of lighting and camera incident angle;

- Strong sun: depending on the incident angle of camera, images may contain bright regions due to the reflection from the insulator.

Fig. 3 shows some example of insulator images under different weather conditions.

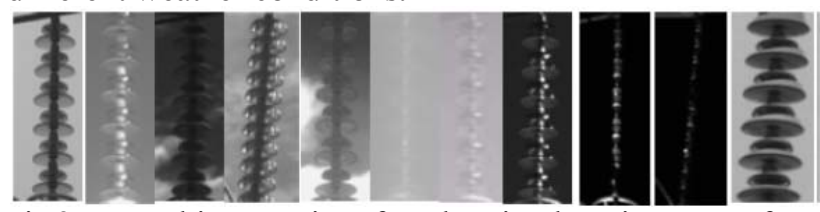

Fig.3 Zoomed in scenarios of outdoor insulator images. Left to right: sunny; sunny with reflection; 3 non-uniform cloudy backgrounds; 2 blurred images due to foggy; 3 images in dark nights; insulator covered with snow.

For detecting and estimating snow/ice coverage and swing angles, several essential steps are involved (i.e., pre-processing, detection, and estimation). Fig.4 shows the block diagram for detecting and estimating snow/ice.
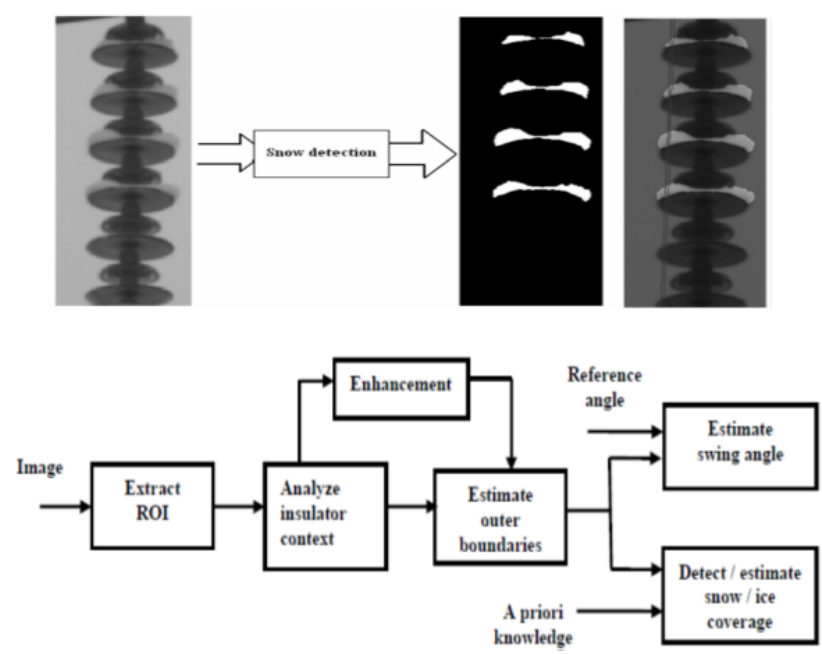

Fig.4 Block diagram of the proposed video surveillance system for snow/ice detection and estimation, and swing angle estimation.

In the pre-processing step, individual insulator image regions (refer to ROI: region of interest, in the text below) are extracted by first using an image registration technique to alignment drifted images, followed by applying vertical and horizontal accumulations of image histogram for dark images and cross-correlations for light 
images. For detection of snow or ice, extra regions above insulator shells are detected through estimating the outer boundary of insulator or silhouette of insulator shells, and comparing with the pre-stored insulator profile, where rigid object assumption is used. For analysis the level of snow/ice coverage, a narrow-width vertical bar, parallel to the vertical center axis of insulator, is swept from the left to the right side of the insulator, where the heights of detected snow regions are accumulated, yielding the maximum and average values of snow/ice coverage for each insulator.

\section{ESTIMATE SWING ANGLES}

Due to the camera view angle, angles of insulator in images do not need to be aligned with vertical axis. The swing angle is hence defined as the relative angle change, computed from the difference between the absolute insulator angle in the given image and the reference insulator angle in an image captured when no wind is present. The first few steps for estimating the angle are the same as snow estimation (see Fig.4). The principle is to compute orientation-related cross-correlations of outer boundaries between the extract ROI image and the reference insulator image (with broadened outer boundaries) over a range of angles through rotating the template, and find the angle associated with the maximum correlation value. Once the absolute angle is estimated, the relative angle is then computed as the swing angle.

\section{INSULATOR VISIBILITY}

For measure the visibility of insulator, similar type of pre-processing is required to extract ROIs containing these insulators through image registration and crosscorrelation. Further, vertical and horizontals lines (socalled "Hough lines") are used to estimate the vertical axis and connecting point of insulators. Additionally, next immediately area to each foreground (FG) insulator region is extracted and used as background (BG) images. Fig.5 shows the automatically detected axis, connect points, and FG/BG regions.

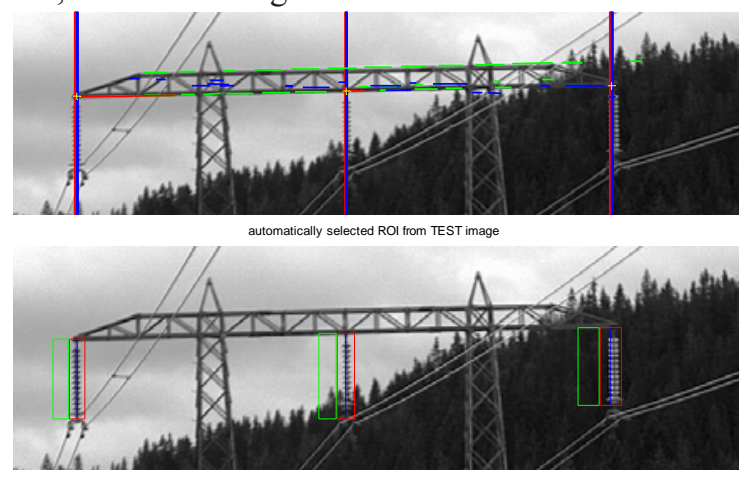

Fig.5 Top: estimated top position of insulator connecting to the tower $($ yellow + ), vertical axis (blue: estimated, red: ground truth), green horizontal lines (from Hough lines around 90 degree). Bottom: Extracted insulator (FG: red box) areas, background (BG: green box) areas, and vertical axis (blue).
Once FG and BG regions extracted for each insulators, visibility measures are defined through exploiting the intensity difference, contrast difference and structure difference between the FG and $\mathrm{BG}$ of each individual insulator, as shown in Fig.6.

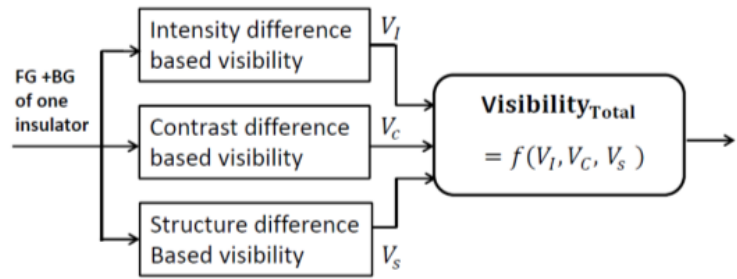

Fig.6 Block diagram for computing insulator visibility based on intensity, contrast and structure differences between the FG and $\mathrm{BG}$ image of each insulator.

Intensity difference-based visibility: According to Ernest Weber, the just noticeable intensity change $\Delta I$ is linearly proportional to the background intensity I, and the ratio $\Delta \mathrm{I} / \mathrm{I}$ is a constant [4]. This implies that with a same amount of intensity change, it is more noticeable if the background is dark than bright. This is taken into account in the visibility measure. Further, as background may contain multiple modes (e.g. dark trees and light sky), considering several modes may lead to more accurate measure. Hence, we first use k-mean clustering to estimate multiple modes (a maximum of 2 modes is used in our tests). We then compute the histogram difference between FG and BG images, and retain those bins where only foreground pixels exist (see the green bin area in the bottom plot of Fig.7). After that, relative intensity difference is computed for each region and averaged over different mode regions weighted by the number of pixels.
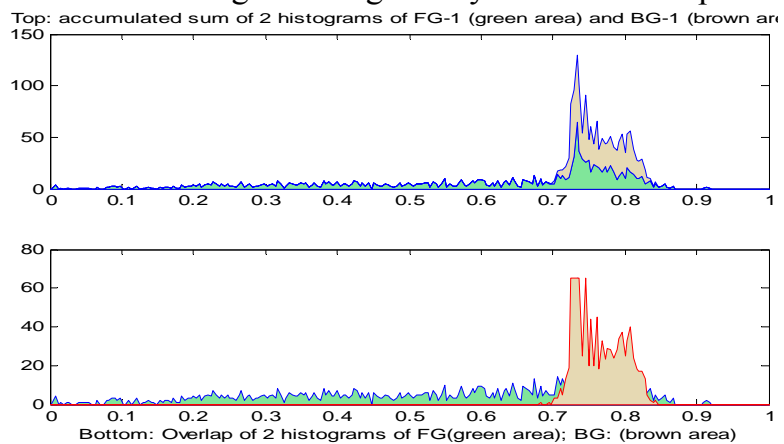

Fig.7 Histograms of FG and BG. Top: histograms from the BG (brown) is added to the FG (green). Bottom: histograms from the BG (brown) and FG (green) overlap each other. Noting: only the green bin part that is larger than brown bin part is considered in the intensity visibility measure.

Contrast difference-based visibility: The definition is similar to the constrast similarity measure in [5], where the difference is that our constrast difference is defined between the FG and $\mathrm{BG}$ regions.

Structure difference-based visibility: For this measure, subband filtered FG and BG images are considered, since image structures are most visible in high frequency 
bands. Further, structures are examined according to statistics in each image [6] rather than pixel-wise comparisons [5]. Our definition is similar to that in [6] however, we only use 2 subband images $\mathrm{I}_{0,1}$ and $\mathrm{I}_{1,0}$ obtained from the $2^{\text {nd }}$ level of wavelet transform and applies to FG and $\mathrm{BF}$ image regions.

Total visibililty measure: is a function of these 3 items. For simplicity, we define it as the product of the above 3 items:

Visibility $_{\text {Total }}=V_{\text {intensity }} * V_{\text {contrast }} * V_{\text {structure }}$

Since the value of 1.0 implies the most similar between the $\mathrm{FG}$ and $\mathrm{BG}$ regions (i.e., the least visible of an insulator), the more visible the insulator, the smaller the value in (1) (smallest value is 0 ).

\section{EXPERIMENTAL RESULTS}

Snow/Ice Detection and Estimation: The automatic image-processing algorithm has been tested over a period of about two years. Fig 8 shows some results (left) and the steps in the test (right). A GUI is also developed for the system [3].
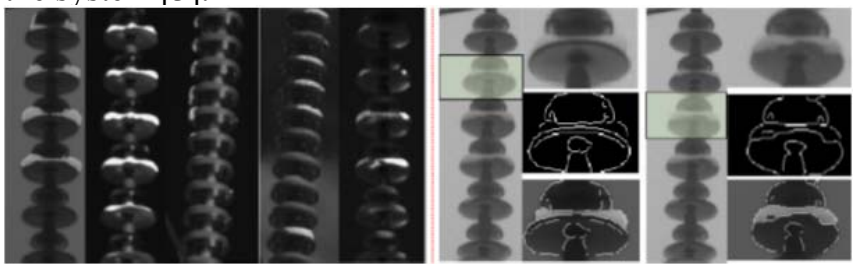

Fig. 8 Left part: automatic analysis results, containing 5 images where detected snow regions are visually enhanced: the first 3 insulators: good results from images with 1 clear and 2 dark background; the next 2 insulators: not so good results where only parts of snow areas are detected from images with cloudy and dark background. Right part: Semi-automatic analysis of snow ground truth for shells 2 and 3 . From left to right, top to bottom in each column: Selecting a shell (inside green box) from ROI; a selected shell, closed edge curves after modification, resulted ground truth snow region.

The performance of automatic analysis results is evaluated for insulator type (b) by comparing with semiautomatically generated 'ground truth' snow area as the reference, summarized in Table I. From Table I, one can see that the average performance is significantly dragged down by poor quality images with low visibility, very weak edges and dark snow in the sets of bright and dark images. The highest snow coverage during that period was $14.73 \%$.

\begin{tabular}{|l|l|l|}
\hline & Detection rate (\%) & False alarm (\%) \\
\hline $\begin{array}{l}\text { Good quality } \\
\text { images }\end{array}$ & 93.00 & 9.00 \\
\hline Bright images & 61.03 & 21.50 \\
\hline Dark images & 74.22 & 18.13 \\
\hline
\end{tabular}

Table I. Performance evaluation from snow/ice detection and estimation algorithms, using images in about one month period

Visibility Estimation: For three insulators, their backgrounds are rather different: pure sky, sky plus forest, pure forest. Fig.9 shows an example of some test results. The objective visibility estimated here is determined by each individual insulator and its background. For comparison of visibility of different insulators, it is desirable that insulators be placed against a same background.

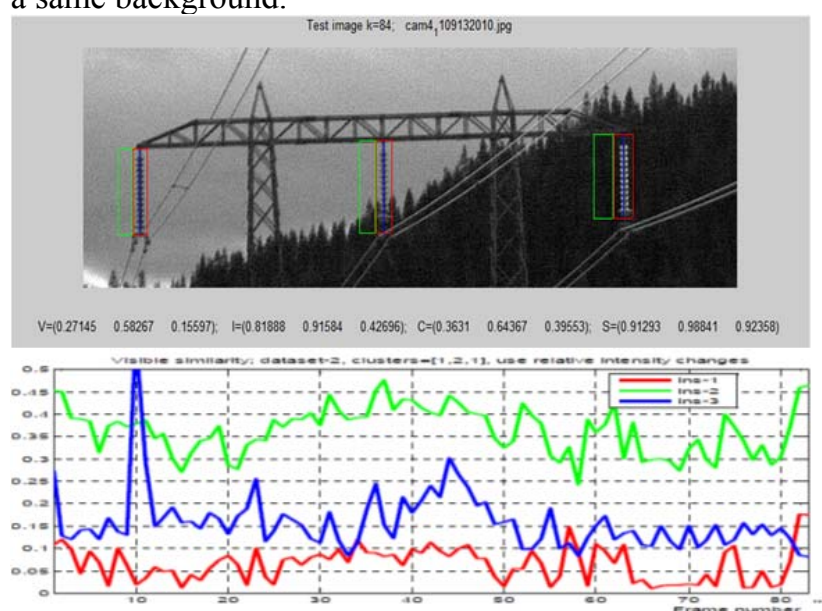

Fig. 9 Test results of insulator visibility. Top: result from one frame image: where the bottom line contains shows the resulting values for the 3 insulators: $\mathrm{V}=\mathrm{V}_{\text {Total }}, \mathrm{l}=\mathrm{V}_{\text {Intensity }}, \mathrm{C}=$ $\mathrm{V}_{\text {Contrast }}$ and $\mathrm{S}=\mathrm{V}_{\text {Structure }}$; Bottom: results as a function of time (over 85 frames) for the 3 insulators with the given backgrounds (pure sky, tree and sky, and pure trees).

\section{CONCLUSION}

This paper has presented the use of image analysis-based methods and video surveillance for extracting information of insulator states in power transmission lines. Three possible applications are presented. The promising results are obtained on automatic detection and estimation of snow/ice coverage, swing angle and objective quantification of visibility of electrical insulators based on image analysis techniques.

It is worth noting that comparing visibility of different insulators, further study is required by using similar backgrounds on a range of lighting conditions especially in scenarios where sun-reflection in insulators is present.

\section{REFERENCES}

[1] WAP project website: http://wap.stri.se

[2] S.M.Berlijn, I.Gutman, et al,"Laboratory Tests and a Web Based Surveillance to determine the Ice- and Snow Performance of Insulators", IEEE Trans. Dielectrics and Electrical Insulation,Vol.14, No.6, pp.1373-1380, 2007

[3] I.Y.H.Gu, U.Sistiaga, S.M.Berlijn, A.Fahlström, "Online detection of snow coverage and swing angles of electrical insulators on power transmission lines using videos", pp.3249-3252, IEEE int'l conf. image processing, 2009.

[4] Weber's law of just noticeable differences: http://people.usd.edu/ schieber/coglab/WebersLaw.html

[5] Z.Wang, A.C.Bovik, et al, "Image quality assessment: from error visibility to structure similarity", IEEE trans. on image processing, pp.1-13, vol.13, No.4, 2004.

[6] X.Zhao, M.G.Reyes, et al, "Structural texture similarity metrics fir retrieval applications", proc. IEEE int'l conf. Image processing, 2008. 